\title{
Use of antiplatelet drugs and the risk of mortality in patients with COVID-19: a meta-analysis
}

\author{
Chia Siang Kow ${ }^{1}\left[\right.$. Syed Shahzad Hasan ${ }^{2,3}$
}

Accepted: 22 March 2021 / Published online: 4 April 2021

( ) The Author(s), under exclusive licence to Springer Science+Business Media, LLC, part of Springer Nature 2021

Globally, there have been close to 130 million reported cases of coronavirus disease 2019 (COVID-19) as of 2nd April 2021, with 2.8 million deaths documented [1]. In order to reduce the risk of mortality associated with COVID19 , several drugs have been repurposed for its treatment. However, a drug with widespread availability around the globe is particularly desirable to be repurposed for the treatment of COVID-19, since it could be immediately trialed in large-scale studies, and immediate access could be guaranteed shall the drug is proven effective to reduce the risk of COVID-19 associated deaths. One of the first drugs to be introduced for routine usage in the medical field - aspirin (also known as acetylsalicylic acid), is still one of the most widely used medications, with an estimated 50-120 billion pills consumed each year [2]. In addition, it is one of the most researched drugs in the world, with an estimated 700-1000 clinical trials conducted annually [3].

A recent appraisal of evidence on the potential effects of aspirin in the context of COVID-19 suggests that aspirin deserves to be tested in patient population with COVID-19, based on the improved survival observed with the use of aspirin among patients with different types of infections, characterized by overactivation of the inflammation cascade and enhanced platelet reactivity [4]. Interestingly, thromboinflammation, which is a term coined to illustrate the coordinated activation of the inflammatory and thrombotic responses, is a major cause of morbidity and mortality in patients with COVID-19 [5]. Indeed, aspirin had been previously reported to reduce the risk of acute respiratory distress

Chia Siang Kow

chiasiang_93@hotmail.com

1 School of Postgraduate Studies, International Medical University, Kuala Lumpur, Malaysia

2 School of Applied Sciences, University of Huddersfield, Huddersfield, United Kingdom

3 School of Biomedical Sciences \& Pharmacy, University of Newcastle, Callaghan, Australia syndrome [6] and its associated mortality [7] in the critically ill non-COVID-19 population, which also happens to be the major complication that arises from thrombo-inflammatory responses in severe cases of COVID-19 with accompanied high mortality rate [8].

While it seems reasonable for aspirin or even antiplatelet agents as a whole to be repurposed for the treatment of COVID-19, the retrospective cohort study by Ho and colleagues [9] failed to demonstrate an association between the use of antiplatelets and improvement in clinical outcomes in patients with COVID-19. The aforementioned study [9] enrolled only patients with COVID-19 from the United States, whereas several studies from different parts of the world have since explored the association between the use of antiplatelets and clinical outcomes in patients with COVID19. Thus, we performed a meta-analysis of the available studies to explore the overall effect of the use of antiplatelets in patients with COVID-19.

We performed a systematic literature search in electronic databases including PubMed, Google Scholar, Scopus, and preprint servers (medRxiv, Research Square, SSRN) with no language restriction for eligible studies published up to February 15, 2021. The search strategy was built based on the following keywords and their MeSH terms: "COVID19", "SARS-CoV-2", "antiplatelet", "aspirin", and "acetylsalicylic acid". Two investigators (CSK and SSH) independently performed literature screening to identify eligible studies. The reference lists of relevant articles were also hand-searched for additional studies. Studies eligible for inclusion were studies with any design that investigated the preadmission/pre-diagnosis or ongoing use of antiplatelet on the risk of a fatal course of COVID-19 and reported adjusted measures of association. We excluded editorials or narrative reviews without original data. In addition, studies that provided no adjusted estimation were also excluded. The quality of observational studies was evaluated using the Newcastle-Ottawa Scale [10], with a score of $>7$ indicating high quality. 


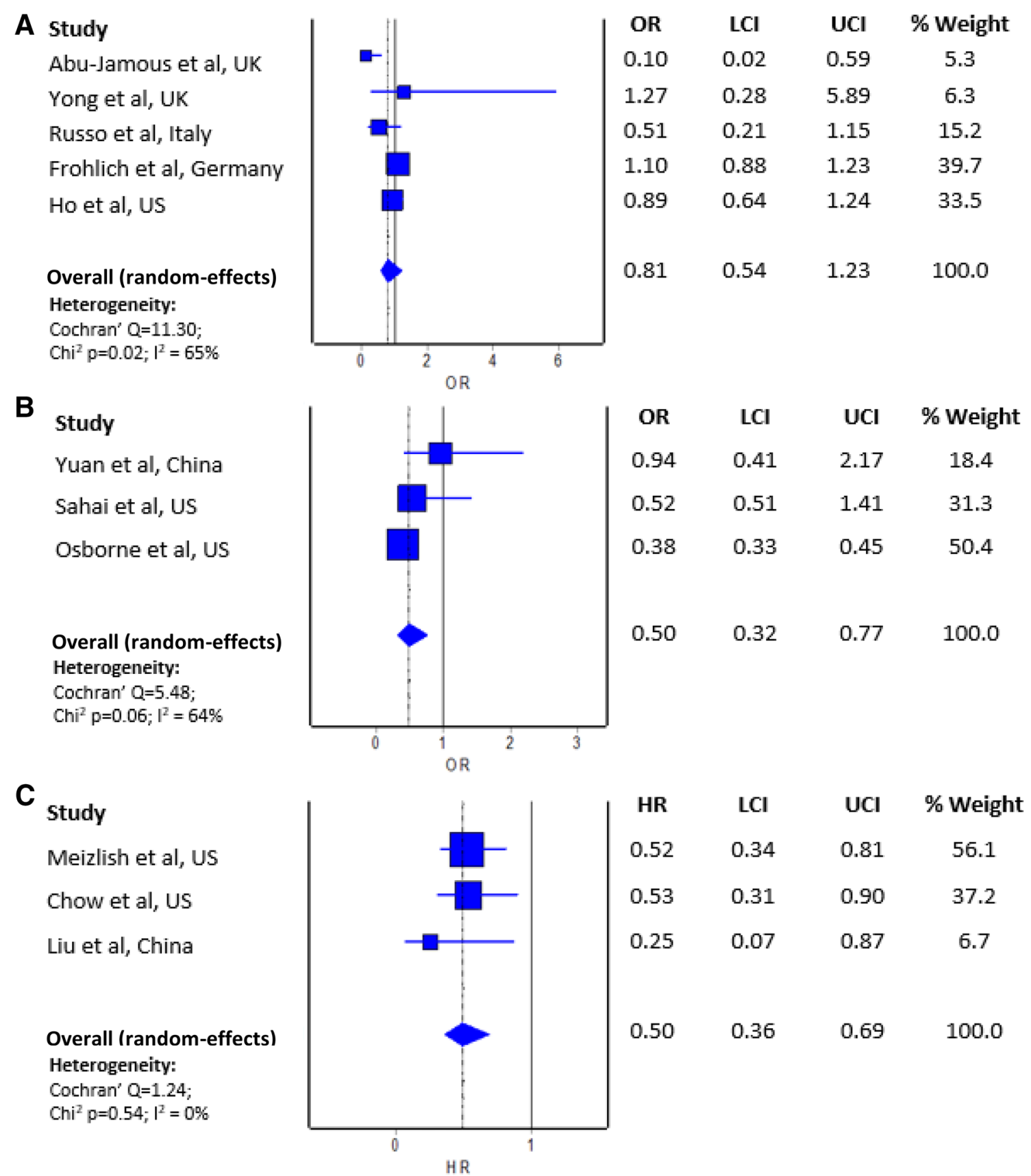

Fig. 1 Forest plot showing the pooled odds ratio (OR) or hazard ratio (HR) of mortality of patients with COVID-19 treated with antiplatelets versus no antiplatelets (a) and with aspirin versus no aspirin $(\mathbf{b}, \mathbf{c})$

The outcome of interest was the development of a fatal course of COVID-19. Each included trial was independently evaluated by two investigators (CSK and SSH) who extracted the study characteristics. Data collected included authors, study design, country, patients' age, the total number of included patients, mortality outcomes, adjusted mortality estimates, and covariates. The disagreement between the two investigators related to the inclusion of studies, extraction of data, and quality appraisal of included studies was resolved through mutual discussions. Adjusted odds ratios or relative risks and the corresponding $95 \%$ confidence intervals (CIs) from included studies were pooled using a random-effects model to produce pooled odds ratio and $95 \%$ confidence interval. We examined the heterogeneity between studies using the $\mathrm{I}^{2}$ statistics with $50 \%$, and using the $\chi^{2}$ test with $\mathrm{P}<0.10$, as the thresholds for statistically significant heterogeneity. All analyses were performed using Meta XL, version 5.3 (EpiGear International, Queensland, Australia). 


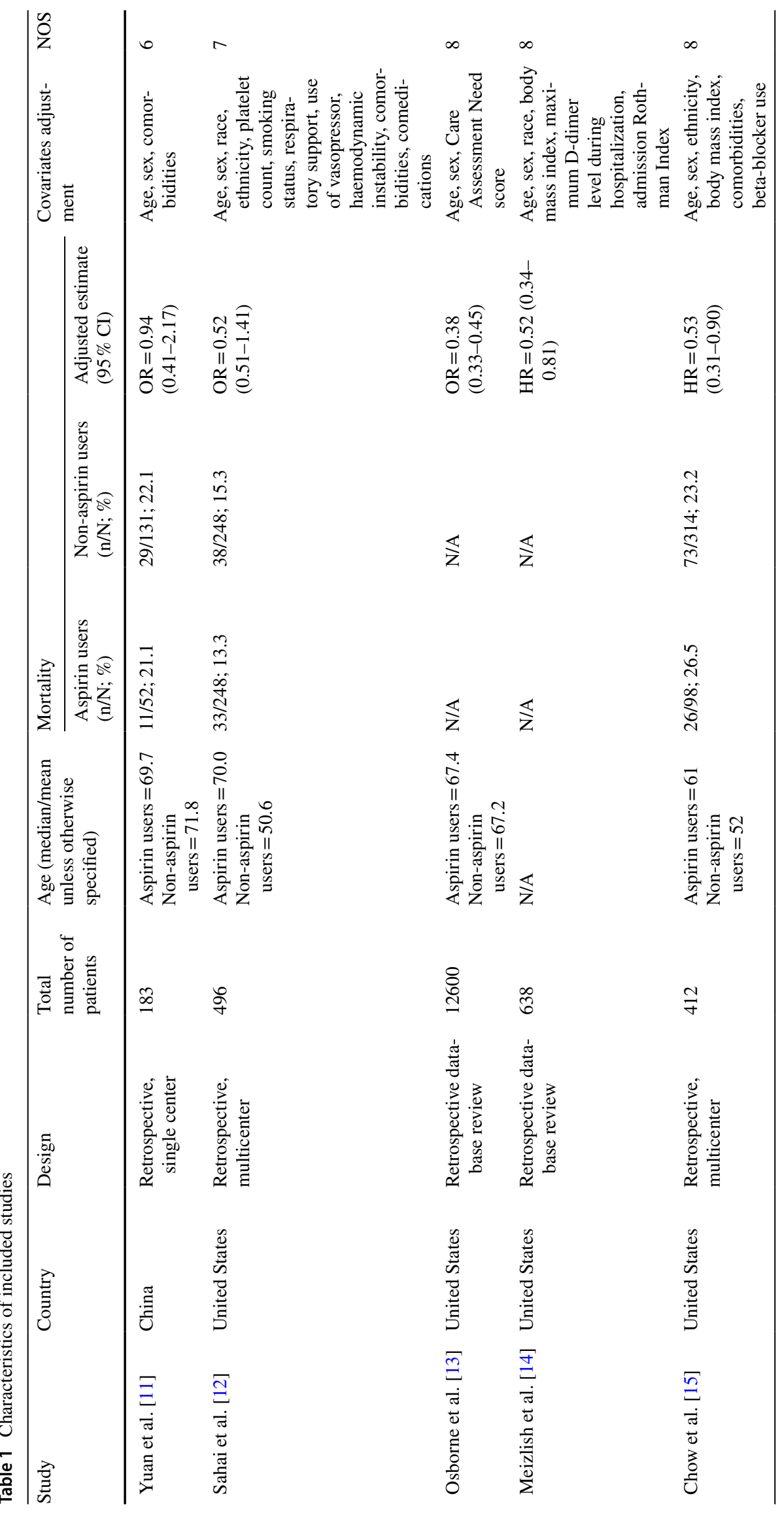




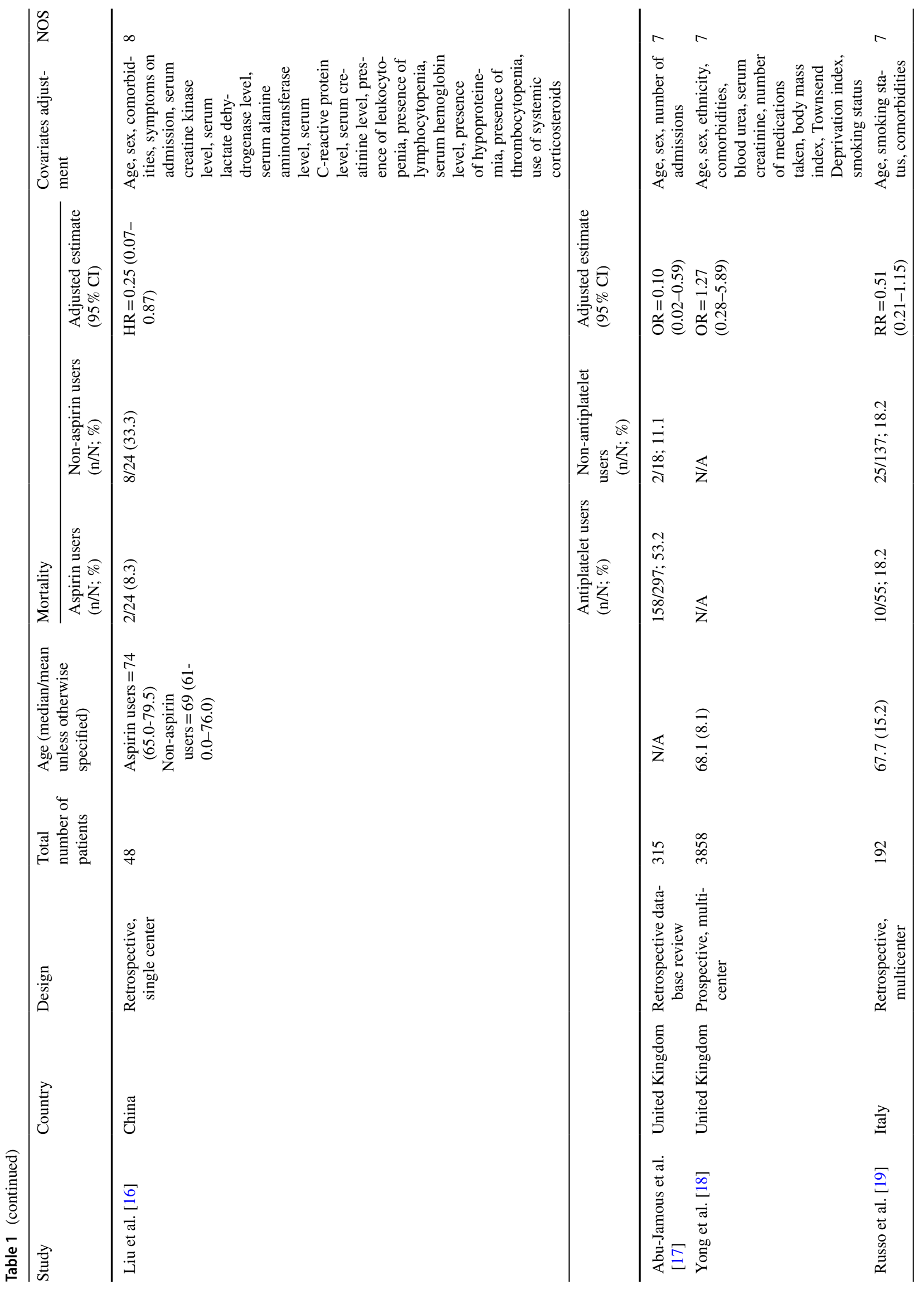




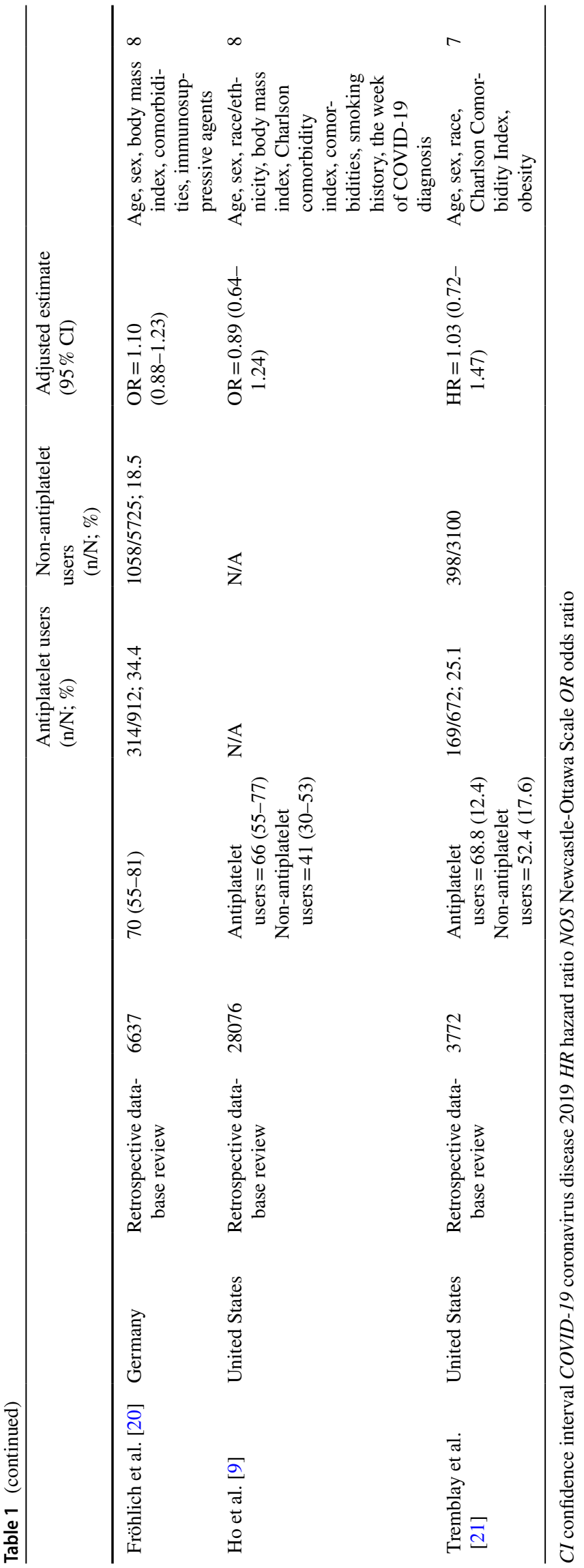

Our literature search yielded 483 unique abstracts. After deduplication and application of the eligibility criteria, eighteen relevant articles were shortlisted for inclusion through full-text examination. Of these, six studies were excluded since they reported no adjusted measures of association. Eventually, 12 studies [9, 11-21] were included for this meta-analysis; 6 studies [9, 17-21] investigated the effect of antiplatelets, and 6 studies [11-16] investigated the effect of aspirin alone. Study characteristics are depicted in Table 1. Across the 6 studies [11-16] which investigated the effect of antiplatelets in patients with COVID-19, all [9, 17, 19-21] but one studies [18] were retrospective in design, with one multicentered study [19] and four database reviews [17, 18, 20, 21]; the remaining one study [18] was a prospective multicentered study. On the other hand, all the 6 studies [11-16] that investigated the effect of aspirin in patients with COVID-19 were retrospective in nature, with two multicentered studies $[12,15]$, two single-centered studies $[11,16]$, and two database reviews $[13,14]$. The included studies were originated from the United Kingdom [17, 18], the United States [9, 12-15, 21], Italy [19], Germany [20], and China $[11,16]$, and they are deemed moderate-to-high quality with Newcastle-Ottawa Scale ranging from 6 to 8 (Table 1).

The meta-analysis of 5 studies [9, 17-20] which reported the effect measures in odds ratio/relative risk revealed no significant difference in the odds for the development of a fatal course of COVID-19 between antiplatelet users and non-antiplatelet users (Fig. 1a; pooled odds ratio $=0.81$; $95 \%$ confidence interval $0.54-1.23$ ). The remaining one study [21] which reported the effect measure in hazard ratio (and thus unable to be included in the meta-analysis) reported consistent findings with the meta-analysis in which no significant difference in the risk for the development of a fatal course of COVID-19 between the two groups (hazard ratio $=1.03 ; 95 \%$ confidence interval 0.72-1.47). However, we observed a significantly reduced risk of a fatal course of COVID-19 with the use of aspirin in patients with COVID-19 relative to non-use of aspirin (Fig. 1b; pooled odds ratio $=0.50 ; 95 \%$ confidence interval $0.32-0.77$ and Fig. 1c; pooled hazard ratio $=0.50 ; 95 \%$ confidence interval 0.36-0.69). These preliminary findings with the use of aspirin in patients with COVID-19 suggest aspirin to be potentially therapeutic in this patient population and support the intention for its repurposing. Although antiplatelet agents have systemic antithrombotic effects, the fact that the use of aspirin was associated with mortality benefits, but not antiplatelet agents as a whole, may be related to aspirin's antiviral effects and anti-inflammatory effects, which are not found in other antiplatelet agents [4]. Nevertheless, the studies included in our meta-analysis are mostly of retrospective design, and thus generalizability of the findings may be limited. There were at least seven ongoing randomized 
controlled trials (Table S1) investigating the mortality outcomes with the use of antiplatelets as standalone or combination therapy in patients with COVID-19 which could clarify their potential risks and benefits in this patient population.

Supplementary Information The online version contains supplementary material available at https://doi.org/10.1007/s11239-021-02436-0.

Funding No external funding was used in the preparation of this manuscript.

\section{Declarations}

Conflict of interest Chia Siang Kow and Syed Shahzad Hasan declare that they have no potential conflicts of interest that might be relevant to the contents of this article.

\section{References}

1. Dong E, Du H, Gardner L (2020) An interactive web-based dashboard to track COVID-19 in real time. Lancet Infect Dis. https:// coronavirus.jhu.edu/map.html. Accessed 26 Dec, 2020

2. Signorelli SS, Platania I, Tomasello SD et al (2020) Insights from experiences on antiplatelet drugs in stroke prevention: a review. Int J Environ Res Public Health 17(16):5840

3. Connelly (2014) A history of aspirin. Pharm J 6(7):20066661

4. Bianconi V, Violi F, Fallarino F, Pignatelli P, Sahebkar A, Pirro M (2020) Is acetylsalicylic acid a safe and potentially useful choice for adult patients with COVID-19 ? Drugs 80(14):1383-1396

5. Gu SX, Tyagi T, Jain K et al (2020) Thrombocytopathy and endotheliopathy: crucial contributors to COVID-19 thromboinflammation. Nat Rev Cardiol 18(3):194-209

6. Boyle AJ, Di Gangi S, Hamid UI et al (2015) Aspirin therapy in patients with acute respiratory distress syndrome (ARDS) is associated with reduced intensive care unit mortality: a prospective analysis. Crit Care 19(1):109

7. Chen W, Janz DR, Bastarache JA et al (2015) Prehospital aspirin use is associated with reduced risk of acute respiratory distress syndrome in critically ill patients: a propensity-adjusted analysis. Crit Care Med 43(4):801-807

8. Hasan SS, Capstick T, Ahmed R et al (2020) Mortality in COVID19 patients with acute respiratory distress syndrome and corticosteroids use: a systematic review and meta-analysis. Expert Rev Respir Med 14(11):1149-1163

9. Ho G, Dusendang JR, Schmittdiel J, Kavecansky J, Tavakoli J, Pai A (2021) Association of chronic anticoagulant and antiplatelet use on disease severity in SARS-COV-2 infected patients. J Thromb Thrombolysis. https://doi.org/10.1007/s11239-021-02383-w
10. Wells G, Shea B, O'Connell D, Peterson J, Welch V, Losos M, Tugwell P (2013) The Newcastle-Ottawa Scale (NOS) for assessing the quality of nonrandomised studies in meta-analyses. http://www.ohri.ca/programs/clinical_epidemiology/oxford. asp. Accessed 26 Dec, 2020

11. Yuan S, Chen P, Li H, Chen C, Wang F, Wang DW (2020) Mortality and pre-hospitalization use of low-dose aspirin in COVID-19 patients with coronary artery disease. J Cell Mol Med. https://doi. org/10.1111/jcmm. 16198

12. Sahai A, Bhandari R, Koupenova M et al (2020) SARS-CoV-2 receptors are expressed on human platelets and the effect of aspirin on clinical outcomes in COVID-19 patients. Preprint. Res Square. rs.3.rs-119031/v1

13. Osborne TF, Veigulis ZP, Arreola DM, Mahajan SM, Röösli E, Curtin CM (2021) Association of mortality and aspirin prescription for COVID-19 patients at the Veterans Health Administration. PLoS ONE 16(2): 0246825

14. Meizlish ML, Goshua G, Liu Y et al (2021) Intermediate-dose anticoagulation, aspirin, and in-hospital mortality in COVID19: a propensity score-matched analysis. Am J Hematol 20:21. https://doi.org/10.1002/ajh.26102

15. Chow JH, Khanna AK, Kethireddy S et al (2020) Aspirin use is associated with decreased mechanical ventilation, ICU admission, and in-hospital mortality in hospitalized patients with COVID-19. Anesth Analg. https://doi.org/10.1213/ANE.0000000000005292

16. Liu Q, Huang N, Li A et al (2021) Effect of low-dose aspirin on mortality and viral duration of the hospitalized adults with COVID-19. Medicine 100(6):e24544

17. Abu-Jamous B, Anisimovich A, Baxter J et al. Associations of comorbidities and medications with COVID-19 outcome: A retrospective analysis of real-world evidence data. Preprint. medRxiv. 2020;2020.08.20.20174169

18. Yong X, Wong KCY, So H. Exploring drugs and vaccines associated with altered risks and severity of COVID-19: a UK Biobank cohort study of all ATC level-4 drug categories. Preprint. medRxiv. 2020;2020.12.05.20244426

19. Russo V, Di Maio M, Attena E et al (2020) Clinical impact of pre-admission antithrombotic therapy in hospitalized patients with COVID-19: A multicenter observational study. Pharmacol Res 159:104965

20. Fröhlich GM, Jeschke E, Eichler U et al (2021) Impact of oral anticoagulation on clinical outcomes of COVID-19: a nationwide cohort study of hospitalized patients in Germany. Clin Res Cardiol. https://doi.org/10.1007/s00392-020-01783-x

21. Tremblay D, van Gerwen M, Alsen M et al (2020) Impact of anticoagulation prior to COVID-19 infection: a propensity scorematched cohort study. Blood 136(1):144-147

Publisher's Note Springer Nature remains neutral with regard to jurisdictional claims in published maps and institutional affiliations. 